\title{
Review Article \\ Emerging Nonvolatile Memories to Go Beyond Scaling Limits of Conventional CMOS Nanodevices
}

\author{
Lei Wang, CiHui Yang, Jing Wen, and Shan Gai \\ School of Information Engineering, Nanchang Hangkong University, Nanchang 330063, China \\ Correspondence should be addressed to Lei Wang; leiwang@nchu.edu.cn
}

Received 4 August 2014; Revised 5 November 2014; Accepted 6 November 2014; Published 23 December 2014

Academic Editor: Xuedong Bai

Copyright (C) 2014 Lei Wang et al. This is an open access article distributed under the Creative Commons Attribution License, which permits unrestricted use, distribution, and reproduction in any medium, provided the original work is properly cited.

Continuous dimensional scaling of the CMOS technology, along with its cost reduction, has rendered Flash memory as one of the most promising nonvolatile memory candidates during the last decade. With the Flash memory technology inevitably approaching its fundamental limits, more advanced storage nanodevices, which can probably overcome the scaling limits of Flash memory, are being explored, bringing about a series of new paradigms such as FeRAM, MRAM, PCRAM, and ReRAM. These devices have indeed exhibited better scaling capability than Flash memory while also facing their respective physical drawbacks. The consequent tradeoffs therefore drive the information storage device technology towards further advancement; as a result, new types of nonvolatile memories, including carbon memory, Mott memory, macromolecular memory, and molecular memory have been proposed. In this paper, the nanomaterials used for these four emerging types of memories and the physical principles behind the writing and reading methods in each case are discussed, along with their respective merits and drawbacks when compared with conventional nonvolatile memories. The potential applications of each technology are also briefly assessed.

\section{Introduction}

CMOS-based data storage devices are currently used in ubiquitous applications in the daily life of global citizens, and these applications range from embedded memories to mass storage systems. As one of the most commonly used CMOS electronic devices, Flash memory has undoubtedly dominated the semiconductor storage market so far because of its ultra-high density, low cost, short data latency, and nonvolatility. In Flash memory, the writing/erasing process is accomplished by injecting electrons into a so-called "floating gate" device or removing electrons from such a floating gate, while readout is realised by sensing the current flowing through a conductive channel formed inside a p-type substrate. According to the difference in cell structure, Flash memory can be categorised into NAND Flash and NOR Flash. NAND Flash allows for a high storage capacity and supports a fast writing/erasing rate and therefore has been widely used for storage applications including memory cards, USB drives, and solid state drives [1]. Compared with NAND Flash, NOR Flash usually gives a faster readout rate but at the expense of storage density. Hence, NOR Flash is often used where fast code execution is required such as in the BIOS of personal computers and finds applications in almost all handheld devices including cellphones and PDAs [2]. However, it should be noted that although the performance of Flash memory has been improved significantly during the last decade with the aid of some innovative scaling technologies, it will inevitably approach its fundamental physical limits. First, the physical scaling size of Flash memory mainly depends on state-of-the-art photolithography techniques. Owing to the diffraction limit, the minimum feature size that can be provided by the conventional photolithography system is confined to approximately $40 \mathrm{~nm}[3,4]$. Second, the tunnel oxide layer inside Flash memory needs to be thicker than $8 \mathrm{~nm}$ in order to eliminate possible electron leakage, thus eroding the scaling margin [5]. Moreover, the gate coupling ratio must be maintained at a value greater than 0.6 so as to control the conductive channel and prevent gate electron injection [6]. This can be achieved by wrapping the control gate around the floating gate to geometrically increase 
the gate coupling ratio. Obviously, adequate space is unavailable to contain such a wrapping structure when the downscaling process continues. Furthermore, a relatively long distance between two adjacent cells inside Flash memory is required to suppress the crosstalk effect that the electrons stored in one cell would start to have on adjacent cells, adversely affecting the performance of scaled devices [7]. Owing to the aforementioned drawbacks, the state-of-the-art NAND and NOR devices are restricted to $20 \mathrm{~nm}$ node [7] and $45 \mathrm{~nm}$ node [6] sizes, respectively.

Considering the difficulty in further scaling Flash memory below $20 \mathrm{~nm}$, researchers have recently started exploring noncharge based semiconductor memories that are inherently immune to the issues Flash memory is currently facing. According to the recording mechanisms, these memories can be divided into ferroelectric random access memory (FeRAM), magnetic RAM (MRAM), phase change RAM (PCRAM), and resistive RAM (ReRAM). The recording function of FeRAM is realised by applying an electric field to a ferroelectric layer to switch its polarisation state. As FeRAM does not require electrons movement against the floating gate, this type of memory consumes ultra-low power consumption against which other semiconductor memories cannot compete [8]. In addition, FeRAM has revealed an excellent endurance of greater than $10^{14}$ cycles, close to that of static RAM (SRAM) and much longer than that of Flash memory [9]. As a result, FeRAM has gained considerable attention for use in embedded memory applications such as railway passes, automobile equipment, and domestic electronic appliances. Nevertheless, the fact that the ferroelectric material in FeRAM will lose its essential ferroelectric characteristic at very small thicknesses seems to be an insurmountable barrier for FeRAM to cross at present, deferring the possibility of FeRAM replacing Flash memory in the immediate future. MRAM, on the other hand, takes advantage of the tunnel magneto-resistance (TMR) effect occurring inside a magnetic tunnel junction (MTJ) that consists of an insulator sandwiched between a fixed layer and a free layer to store data. Depending on the relative magnetisation orientations between the fixed layer and the free layer, the MTJ can be switched between a high-resistance state and a low-resistance state, thus denoting binary bits. MRAM is regarded to be the closest to a "universal" memory, because of its ultra-fast speed (around $10 \mathrm{~ns}$ ) and extremely high endurance $\left(>3 \times 10^{16}\right)[10,11]$. A major challenge is the higher current density required to switch the magnetisation orientation along with the scaling process, resulting in extra power consumption. Besides, further scaling the memory cells would make MRAM more prone to thermal noise. The most advanced MRAM today has been limited to 32 nm technology [12] and it is not expected that MRAM can replace NAND Flash in a short time. Inside PCRAMs, binary data are represented by a highly conductive crystalline phase and a highly resistive amorphous phase. The phase transformation in PCRAM devices from either the amorphous state to the crystalline state or vice versa can be accomplished by adjusting the characteristics of the applied current pulse such as the magnitude and the leading/trailing time.
The performance superiority of PCRAM to its alternatives arises from its simple cell structure and low voltage operation, making PCRAM attractive for embedded memory applications. However, the interaction of phase change materials with electrodes may give rise to long-term reliability issues and limit the cycling endurance. In spite of these drawbacks, PCRAM has been used in feature phones to replace NOR Flash since 2011 and in volume production at the $45 \mathrm{~nm}$ node since 2012 [13]. Furthermore, the recent progress in PCRAM technology has clearly demonstrated the excellent scaling potential to and beyond the $5 \mathrm{~nm}$ generation [14]. ReRAM is a newer memory device compared to the aforementioned memories. Although ReRAM can be classified into several groups in terms of the resistive materials used, the fundamental mechanism for storing data is to toggle the resistive material inside ReRAM between a high-resistance state and a low-resistance state according to an appropriate voltage or current. The recent findings that include the introduction of the crossbar structure $[9,15]$ and the selection of suitable switching and electrode materials $[9,16]$, integrated with the improved switching speed of RRAM using $\mathrm{TiO}_{2}[9,17]$, have demonstrated the feasibility of fabricating ReRAMs with ultra-high density, good stability, and fast switching speed, thus making ReRAM a formidable competitor of other next-generation nonvolatile memories. However, the resistive switching mechanism of ReRAM still remains unclear, and is likely to prevent any significant scaling of ReRAM in the near future.

As described above, these new types of nonvolatile memories indeed display superior characteristics to Flash memory in some respects and thus may find more extensive use in applications if their respective physical drawbacks can be overcome by innovative scaling technologies. However, considering the overall performance, the dominant role of Flash memory in the semiconductor storage devices market appears unshakable and not under any serious threat from its rivals as of now. Under this circumstance, in order to break the scaling limits of Flash memory and thus to achieve further dimensional and functional scaling of nonvolatile memories, some novel concepts of nonvolatile memories, including carbon memory, Mott memory, macromolecular memory, and molecular memory, have emerged. Nevertheless, research efforts dedicated to these new types of memories at present are inadequate. Obviously, compared to the conventional memories currently in wide use, these memories are still in a nascent stage and, consequently, their more advantageous performance compared to the performance of conventional CMOS devices is yet to be established. However, it is too early to infer that these memories cannot compete with the conventional devices, especially because comprehensively technical exploration on the corresponding physical principles as well as the likely storage potential of these new devices is yet to be conducted. In order to help understanding the physical mechanisms behind these prospective memories and thus infuse more enthusiasm into the research on the improvement and optimisation of device performance, a detailed review concerning the current status of these emerging technologies becomes crucial. 


\section{Carbon-Based Memory}

Carbon is a very important chemical element for today's science and technology. Depending on different atomic bonding configurations (i.e., $\mathrm{sp}^{1}, \mathrm{sp}^{2}$, and $\mathrm{sp}^{3}$ ), carbon can be classified into several allotropes of which the well-known groups are diamond, graphite, and amorphous carbon. Because of the different atomic configurations, the three allotropes of carbon exhibit distinct physical properties. Diamond has a wide $5.5 \mathrm{eV}$ band gap, the highest hardness for any bulk material, the largest room temperature thermal conductivity, the smallest thermal expansion coefficient, and the largest limiting electron and hole velocities for any semiconductor [18], owing to its strong, directional $\sigma$ bond [19]. Such properties have made cutting and polishing tools as the major industrial applications of diamond. Graphite has strong intra-layer $\sigma$ bonding and weak van der Waals bonding between its layers. As graphite is the most stable form of carbon under standard conditions, many disordered forms of carbon have structures based on its lattice [19]. Unlike diamond and graphite, amorphous carbon can be divided into several groups based on the ratio of $\mathrm{sp}^{3}$ to $\mathrm{sp}^{2}$ bonding and the amounts of hydrogen and nitrogen content. One typical member of the amorphous carbon group is diamond-like carbon (DLC) that has a high fraction of diamond-like $\left(\mathrm{sp}^{3}\right)$ bonds. DLC is a metastable form of amorphous carbon that can be deposited at room temperature. It attains a set of advantageous properties such as high mechanical hardness, large optical transparency, and good chemical inertness [18]. This is driving DLC films to be implemented as protective coatings in various products such as magnetic storage disks, optical windows, biomedical coatings, and micro-electromechanical devices (MEMs) [20]. In addition to the aforementioned allotropes, another allotrope that needs particular attention is graphene that can be described as a one-atom thick layer of graphite. In graphene, carbon atoms are densely packed in a regular $\mathrm{sp}^{2}$-bonded atomic-scale hexagonal pattern, forming a very thin and nearly transparent sheet. Because of its superior stability and efficient conduction of heat and electricity, the primary applications of graphene are currently in the fields of electronics, biological engineering, filtration, photovoltaics, and energy storage.

In addition to the applications mentioned above, the idea of using carbon-based materials for data storage applications has recently been proposed [21-27]. Such a carbon-based memory concept stems from the fact that carbon can be switched between a conductive $\mathrm{sp}^{2}$-rich state and a resistive $\mathrm{sp}^{3}$-rich state by applying an appropriate voltage/current pulse. In principle, the conductive state is achieved when the resulting current density inside the resistive carbon is above the threshold value, while the resistive state can be achieved by acquiring a sufficiently high current density inside the conductive carbon, followed by rapid cooling. In this case these two states can be used to represent binary bits. In contrast to other switchable memory materials, the switching process inside carbon-based memory is fully governed by the bond-reorganisation process, and therefore carbon-based memory exhibits a superior scaling potential to other memory devices because of its inherent mono-atomic nature. Moreover, such a bond reconfiguration is a very fast and stable process, implying that carbon-based memory can have a fast speed as well as a long data retention time. Finally, the high resilience of carbon to a variety of external stimuli would ensure robustness and endurance of such carbon-based memories. The ultra-large storage density and long retention time obviously make carbon-based memories, suitable for archival storage as a replacement for magnetic disks and magnetic tapes, while the fast switching speed and the long endurance make it the most competitive rival against SRAM. It is in this context that carbon materials offer an exciting option of realising future generations of highperformance, cost-effective, and environmentally friendly nonvolatile data storage.

The advent of carbon-based memory can be linked to the finding of the electrical switching phenomenon in grapheme-based material [22]. This study triggered the concept of carbon-based memory that was experimentally accomplished for the first time by Kreupl et al. in 2008 [23]. In Kreupl's experiment, electrical switching of graphene-like conductive carbon and insulating carbon as potential storage materials for high density nonvolatile memory applications has been investigated, respectively, based on different cell structures, as shown in Figure 1.

It was reported that the switching from a low resistive state (LRS) to a high resistive state (HRS) can be achieved using a $8.5 \mathrm{~V}$ pulse for a cell length of $50 \mathrm{~nm}$, while the transformation from HRS to LRS can be realised using a $1.5 \mathrm{~V}$ pulse for a cell with $150 \mathrm{~nm}$ diameter bottom contact and $8 \mathrm{~nm}$ thick insulation carbon [23]. However, the cell architecture adopted by Kreupl et al. for the switching from HRS to LRS is different from the one from LRS to HRS, which is obviously not viable for practical applications. In this case, various forms of carbon such as amorphous carbon, DLC carbon, and graphene were explored for use in memory applications so as to improve Kreupl's original design. In 2010, the nonvolatile resistive switching phenomenon was observed in hydrogenated amorphous carbon $(\mathrm{a}-\mathrm{C}: \mathrm{H})$ by Zhuge et al. [24]. The designed carbon memory that consists of a hydrogenated amorphous carbon film sandwiched by a copper layer and a platinum layer $(\mathrm{Cu} / \mathrm{a}-\mathrm{C}: \mathrm{H} / \mathrm{Pt})$ allows for a device yield of $90 \%$, an ON/OFF ratio $>100$, and a retention time $>10^{5} \mathrm{~s}$. Chai et al. replaced the metal bottom electrode with carbon nanotube (CNT) electrode to form amorphous carbon-based memory with an $\mathrm{Au} / \mathrm{a}-\mathrm{C} / \mathrm{CNT} / \mathrm{a}$ $\mathrm{C} / \mathrm{Au}$ configuration [25]. It was reported that such a structure can effectively reduce the size of the active device area as well as the writing current. In addition, a resistance RAM device based on the structure $\mathrm{Pt} / \mathrm{DLC} / \mathrm{W}$ has been proposed by Fu et al. [26]. This DLC based memory exhibited excellent memory performance such as a high ON/OFF resistance ratio $(>300)$, high switching speed $(<50 \mathrm{~ns})$, low operation voltage $(<1.2 \mathrm{~V})$, low switching power consumption $(<16 \mu \mathrm{W})$, nondestructive readout, and good reliability. More recently, Dellmann et al. have developed hydrogenated amorphous carbon-based memory with a $\mathrm{Cu} / \mathrm{a}-\mathrm{C}: \mathrm{H} / \mathrm{TiN}$ structure, resulting in a high ON/OFF resistance ratio (>1000), high switching speed $(<30 \mathrm{~ns})$, and nondestructive readout [27]. 


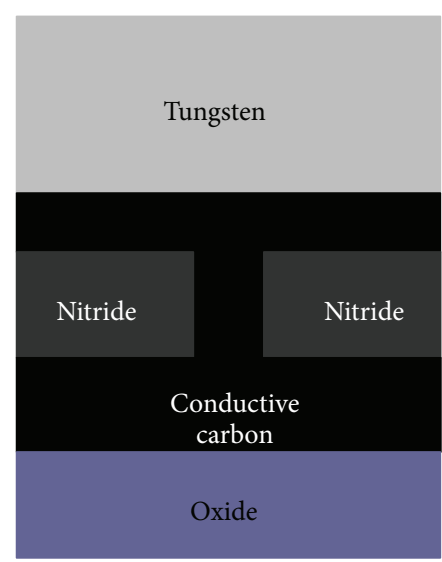

(a)

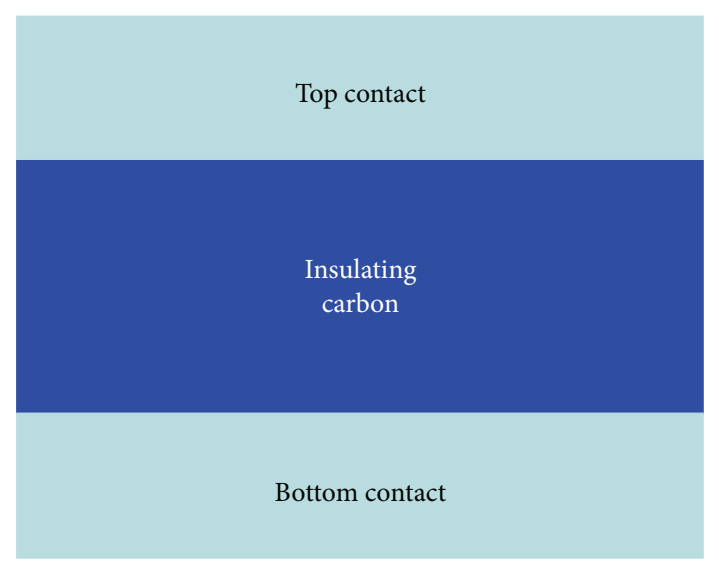

(b)

FIgURE 1: The carbon-based memory cell based on (a) conductive carbon and (b) insulating carbon.

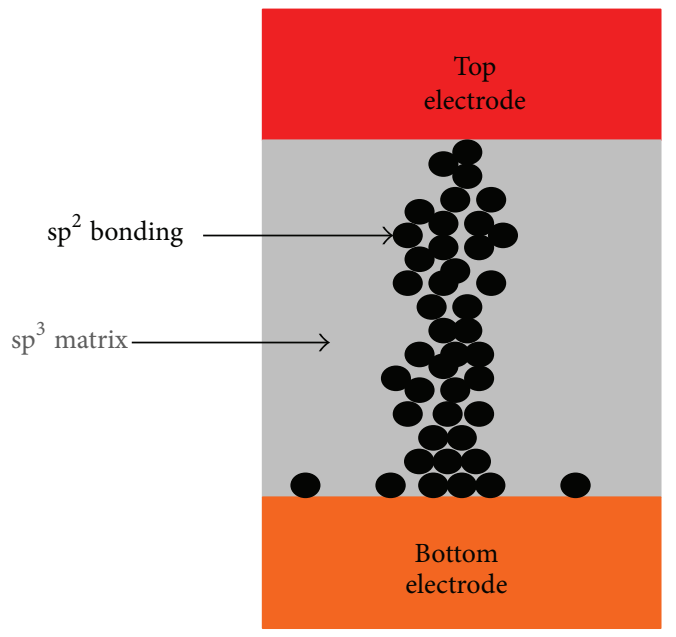

(a)

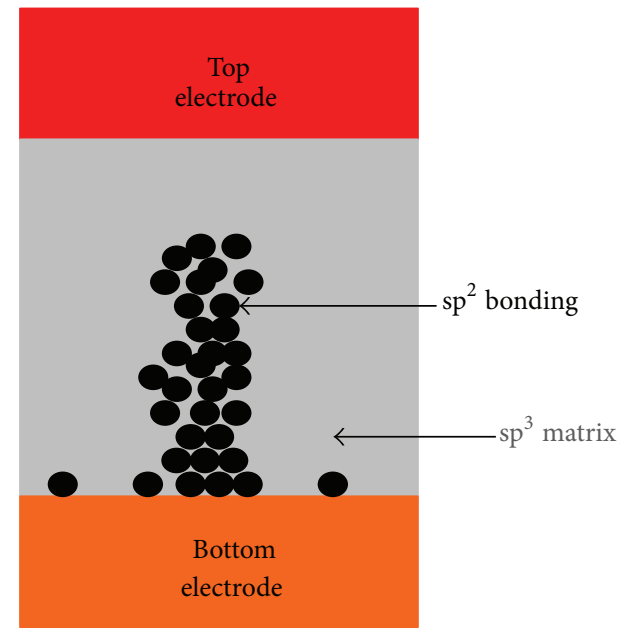

(b)

FIGURE 2: "ON" and "OFF" states of carbon-based memory induced by (a) the formation of a conductive filament and (b) the rupture of a conductive filament.

Although carbon-based memories have recently elicited increased interest, the exact physical mechanism causing this resistive switching in carbon materials is not clear. At present, the consensus is that such a switching behaviour is mainly governed by the formation and rupture of the $\mathrm{sp}^{2}$ (graphitelike low resistive state) filaments inside the $\mathrm{sp}^{3}$ (diamond-like high resistive state) matrix [28], as shown in Figure 2.

The detailed process of $\mathrm{sp}^{2} / \mathrm{sp}^{3}$ conversion still is not well understood. Some studies have ascribed the $\mathrm{sp}^{2} / \mathrm{sp}^{3}$ conversion to the thermal effect $[27,28]$. During the SET process, a sufficiently high voltage is applied into the insulated layer to generate a high tunnelling current. The Joule heating effect resulting from the tunnelling current would help atoms to gain adequate kinetic energy to overcome the energy barrier between LRS and HRS, thus generating the clustering of $\mathrm{sp}^{2}$ hybridised carbon atoms along the temperature gradient. This will lead to a formation of graphitic filaments extending through the entire cell thickness, resulting in LRS. It was suggested that the graphitic filament will reach the maximum growth rate at a temperature of $2400 \mathrm{~K}$. At a lower temperature, the atom cannot gain enough kinetic energy to overcome the energy barrier, whereas at a higher temperature, the kinetic energy of carbon atoms would be too high to maintain their positions at LRS. For the RESET process, a large voltage pulse with a short duration is applied into the conductive filament. Once the peak temperature at the central part of the filaments has exceeded the melting point of graphite, the filament will undergo a rupture (melting) at the central region in a very short time. After the removal of the voltage, the temperature falls back to room temperature, resulting in HRS. Besides the thermal induced transition, some researchers have proposed a field-dependent hypothesis to interpret the $\mathrm{sp}^{2} / \mathrm{sp}^{3}$ conversion $[21,29]$. According to this hypothesis, the reaction rate from $\mathrm{sp}^{3}$ to $\mathrm{sp}^{2}$ strongly depends on the electric field intensity, while the reaction rate from $\mathrm{sp}^{2}$ to $\mathrm{sp}^{3}$ is primarily determined by the thermal stress effect. In the SET 
process, the initial HRS of the device leads to a small electric current and a high electric field. Once the resulting electric field intensity exceeds a threshold, the reaction rate from $\mathrm{sp}^{3}$ to $\mathrm{sp}^{2}$ will increase, leading to LRS. In the RESET process, the applied high voltage would generate a large temperature gradient induced in the region near the conductive filament, thus giving rise to the transition from $\mathrm{sp}^{2}$ to $\mathrm{sp}^{3}$. Recently, a hydrogen-induced redox model has been presented as a likely mechanism responsible for the resistive switching of hydrogenated amorphous carbon [30]. This model infers that during the RESET process, hydrogen atoms are pulled from the top electrode and absorbed by the double bonds of $\mathrm{sp}^{2}$ carbon, the so-called hydrogenation process. Such a reaction will break the conductive $\mathrm{sp}^{2}$ filament into the resistive $\mathrm{sp}^{3}$ matrix. During the SET process, the applied voltage can significantly lower the barrier through the hopping conduction process, and in this case the hydrogen atoms are attracted toward the top electrode to form the conjugation double bonds of the $\mathrm{sp}^{2}$ filament, called the dehydration process. In contrast to the $\mathrm{sp}^{2} / \mathrm{sp}^{3}$ conversion, the formation of metal ions filament inside carbon materials is considered to be responsible for the resistive switching. A positive voltage is briefly applied during the SET stage to the top metal electrode to drive metal ions into the carbon-based materials, giving rise to a conductive filament. During the RESET stage, a sufficiently high voltage of opposite polarity is applied to induce the electrochemical dissolution of the metal filaments to achieve the highly resistive state. As material parameters and experimental environments are different within these studies, the physical reality of these inferences still need to be further verified, thus necessitating the exploration of more plausible interpretations.

\section{Mott Memory}

Mott memory can be regarded as a type of ReRAM that has a different switching mechanism from conventional resistive memories. The cell of Mott memory usually comprises a correlated electron insulator (also called Mott insulator)whose phase can be toggled between a metallic state and an insulated state (Mott transition) - sandwiched by a metal top electrode and a metal bottom electrode, as illustrated in Figure 3.

Therefore, the two resulting resistive states can be used to achieve the data storage functionality. Depending on the specific materials used for Mott memory, the switching mechanism can be attributed to either the electric field-induced effect or the thermal induced effect. The field-dependent mechanism attributes the resistive transition to the dielectric breakdown of the charge-ordered state where the charge carriers are initially localised at each atomic site owing to the electron-electron repulsive interaction, particularly for Mott insulators [31]. Such a dielectric breakdown behaviour can subsequently induce the presence of a metallic state with a large number of mobile charge carriers, intrinsically different from conventional semiconductors or band insulators. This field-induced Mott transition has been found in a correlated electron material of $\operatorname{Pr}_{1-x} \mathrm{Ca}_{x} \mathrm{MnO}_{3}$ [31]. Such a finding has

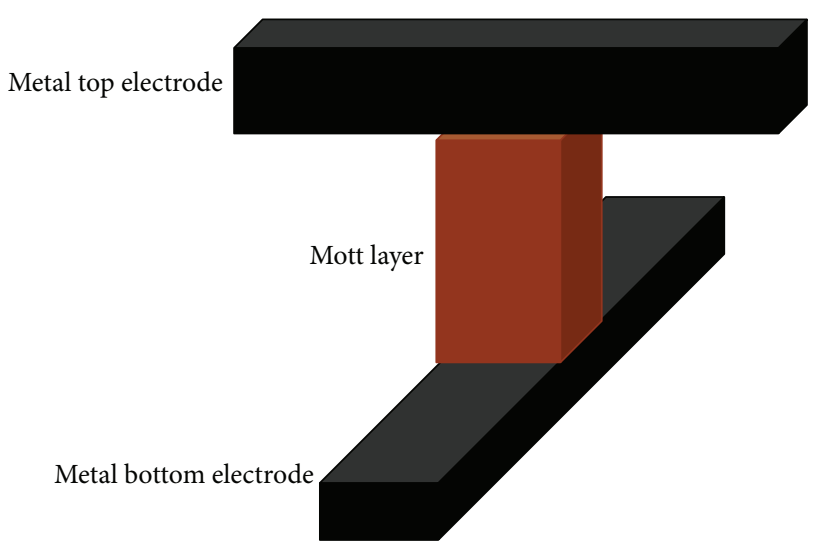

Figure 3: Structure of the Mott memory cell.

resulted in the fabrication of some appealing two-terminal memories utilising these correlated electron oxides such as $\mathrm{VO}_{2}$ [32-34], $\mathrm{SmNiO}_{3}$ [35], $\mathrm{NbO}_{2}$ [36, 37], $\mathrm{Ca}_{2} \mathrm{RuO}_{4}$ [38], and $\mathrm{NiO}[39,40]$. Recently, a new type of Mott memory based on Mott insulator compounds $\mathrm{AM}_{4} \mathrm{X}_{8}(\mathrm{~A}=\mathrm{Ga}, \mathrm{Ge}$; $\mathrm{M}=\mathrm{V}, \mathrm{Nb}, \mathrm{Ta} ; \mathrm{X}=\mathrm{S}, \mathrm{Se}$ ) has been demonstrated to exhibit a nonvolatile characteristic with fast writing/erasing times (50 ns to $10 \mu \mathrm{s}$ ) and resistance ratios of $\Delta R / R$ of the order of $25 \%$ at room temperature [41, 42]. In addition to the two-terminal devices, a three-terminal device, that is, fieldeffect transistors (FETs), made of $\mathrm{VO}_{2}$, has been reported to exhibit a nonvolatile behaviour with provision of a promising prospect for nonvolatile memory applications [43].

The temperature driven Mott transition derives from the fact that the scope of the transitional region initially stemming from the Joule heating above the transformation temperature will be expanded along with the subsequent accumulation of the current flowing into this region, thus causing a Joule heating induced filament $[36,37]$. The Mott transition induced by temperature has been experimentally demonstrated in $\mathrm{NbO}_{2}$ that is subjected to a transformation from a low temperature insulator phase to a high temperature metal phase. The design memory with a size of $110 \times 110 \mathrm{~nm}^{2}$ allows for a fast switching speed of $<2.3 \mathrm{~ns}$ and a switching energy of the order of $100 \mathrm{fJ}[36,37]$. The main challenge of this $\mathrm{NbO}_{2}$-based Mott memory is its volatility due to its thermal induced mechanism.

As Mott memory exhibits a fast switching speed and ultra-low power consumption, the possible applications of Mott memory include smart cards, ID cards, and other embedded memory applications such as railway passes, automobile equipment, and domestic electronic appliances. However, the retention of Mott memory still remains questionable because disorders, defects, and spatial variation of chemical composition may have a remarkable impact on the Mott transition [44-46]. For instance, the Mott transition can be driven even by a small amount of carrier doping to the integer-filling or half-filling valence states of transition element. In this case, the control of crystallinity and the chemical composition in the thin films of correlated electron materials are crucial. Moreover, the existence of the disorder, 


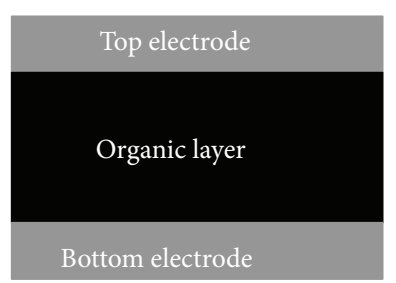

(a)

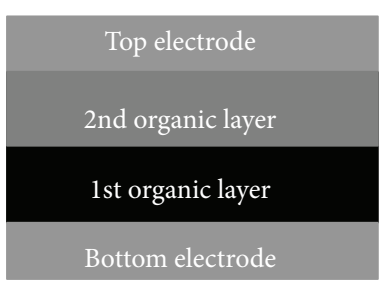

(b)

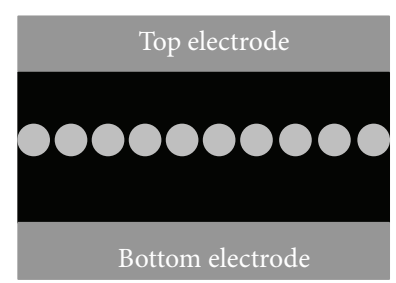

(c)

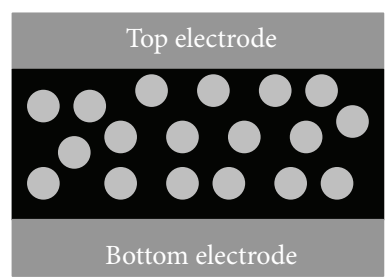

(d)

FIGURE 4: Typical structures of resistive organic memory cell: (a) a single-layer structure without nanoparticles (NPs), (b) a bilayer structure containing two kinds of polymers, (c) a structure with nanotraps buried in the middle of an organic layer, and (d) a polymer-NP composite with NP raps randomly distributed throughout the entire host polymer. Reprinted with permission from [48].

defects, and spatial variation of the chemical composition would require a large amount of carriers of more than $10^{22} \mathrm{~cm}^{-3}$ to induce the Mott transition in actual correlated electron materials, consequently bringing about a fairly large switching voltage [47]. This clearly implies that the interface between the metal electrode and the correlated electron material needs to be controlled precisely.

\section{Macromolecular Memory}

Macromolecular memory is another type of nonvolatile memory that uses polymers as the storage media. The polymer medium used for macromolecular memory whose phase can be switched between a high-resistance state and a lowresistance state contains mainly carbon atoms and is largely amorphous. The cell of macromolecular memory is very similar to Mott memory, making use of a metal/polymer/metal structure. As can be seen from Figure 4, four device structures have been in particular utilised by macromolecular memory [48], that is, a single layer with only one type of polymer material, a bilayer with two types of materials, a trilayer consisting of a nanotraps for charge carriers sandwiched by two polymer layers, and spin-cast polymer-NP blends having a random distribution of nanotraps throughout the entire region of the host matrix.

The physical mechanisms governing the switching behaviour of macromolecular memory are still not well understood, and various explanations have been proposed depending on the structure of the polymer. It is mostly believed that the resistive switching of a poly(3-hexylthiophene) ( $\mathrm{P} 3 \mathrm{HT}$ ) layer sandwiched by copper and aluminium electrodes is induced by the formation of a conductive filament due to the migration of $\mathrm{Cu}$ ions into the organic layer, which was observed by secondary ion mass spectroscopy analysis [49]. Based on this theory, the bias voltage will drive $\mathrm{Cu}$ ions from the anode into the polymer layer and finally towards the cathode. Once the $\mathrm{Cu}$ ions reach the cathode, continuous precipitation of $\mathrm{Cu}$ ions over the cathode occurs, thus resulting in a metallic filament across the entire polymer layer. This will correspond to the "ON" state. Such a filamentary conduction mechanism is also observed in poly(ethylenedioxythiophene). Besides the filamentary type conduction, space-charge-limited conduction (SCLC) is also responsible for the switching mechanism of some polymer materials such as polystyrene (PS)+Au NPSs, and poly[3-(6-methoxyhexyl)thiophene](P30Me) [50] achieved by means of adjusting the interface resistance, while the resistive switching inside the [6,6]-phenyl-C61-butyric acid methyl ester (PCBM) and tetrathiafulvalene (TTF) dispersed in a polystyrene matrix [51] is attributed to the charge transfer effect. It should be noted that the mechanism and its relationship with the polymer structure seem clear in some cases, whereas the mechanism of operation remains largely vague in many cases.

The researchers worldwide are currently focussing on macromolecular memory owing to its fast switching speed and ultra-low power consumption. At the time of writing, a macromolecular memory cell with a very low power consumption $(70 \mathrm{nW})$, a short switching time $(15 \mathrm{~ns})$, and a fairly good stability $\left(85^{\circ} \mathrm{C}, 2 \times 10^{4} \mathrm{~s}\right)$ have been demonstrated [52], implying a ultra-low write energy of $6 \times 10^{-15} \mathrm{~J} / \mathrm{bit}$. A macroscopic memory cell that has a programming voltage of $+1.4 \mathrm{~V}$ and $-1.3 \mathrm{~V}$ for the two states with a good retention time of $>10^{4} \mathrm{~s}$ has recently been reported [53]. Furthermore, using carbon nanotubes as macromolecular electrodes and aluminium oxide as the interlayer, isolated, nonvolatile, rewriteable memory cells with an active area of approximately $36 \mathrm{~nm}^{2}$ has been reported, allowing for a write power less than $100 \mathrm{~nm}$ with an estimated write energy below $10 \mathrm{fJ}$ per bit $[54,55]$. In addition to the conventional polymer media, more recently ferroelectric polymers have been shown to be feasible for the resistive memory applications and could benefit enormously from the development of new macromolecular polymeric materials with combined ferroelectric switching and semiconducting structural units [56]. Therefore, these interesting findings have excited the interests in applications of macromolecular memory for use in portable electronic devices such as PDAs, digital cameras, and mobile phones. The major challenge of the macromolecular memory arises from its poor scalability. To the best of the authors' knowledge, the scalability of macromolecular memory is restricted to $100 \mathrm{~nm}$ at present, far behind the scalability of Flash memory. The desired density improvement mainly depends on a thorough understanding of the switching mechanism occurring inside macromolecular memory. Distinguishing different mechanisms and evaluating the potential and possibilities of each mechanism will therefore be the uppermost priority for optimising the performance of macromolecular 


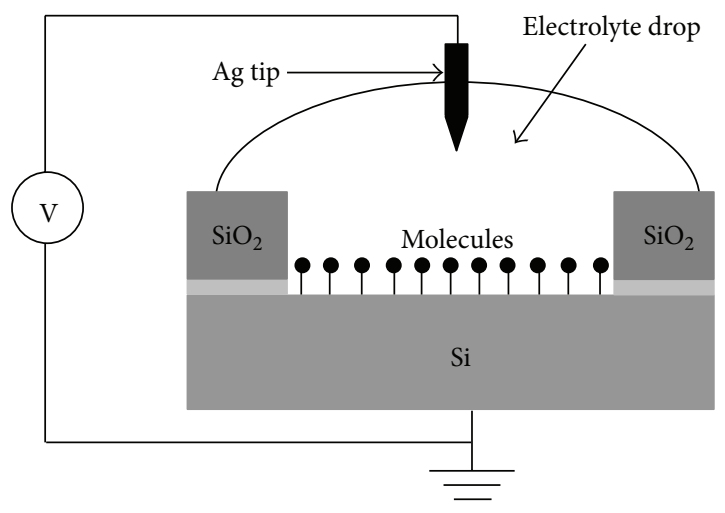

FIGURE 5: Schematic of the capacitive organic-based molecular memory. Reprinted with permission from [60].

memory. Building model systems for each mechanism will also be equally important. Having such model systems would provide an opportunity to benchmark the operation of macromolecular materials. Establishing and securing the collaboration of the chemical industry is indispensable in order to achieve these research targets. More importantly, for the design, synthesis, and development of next-generation macromolecular materials for memory applications, clear guidelines on the required structural and electronic properties of macromolecular materials are needed.

\section{Molecular Memory}

The name "molecular memory" obviously indicates the use of molecular species as data storage media rather than inorganic materials. The storage function of molecular memory is based on molecular switching that can be induced by the change in either the capacitance or the resistance of the cell. In a perfect molecular memory device, each individual molecule contains a bit of data, leading to massive data capacity. Nevertheless, practical devices are more likely to use large numbers of molecules for each bit. Based on the switching mechanism, the molecular memory can be categorised into capacitive molecular memory and resistive molecular memory. The storage mechanism of capacitive molecular memory is strongly dominated by the capture/release of the charge inside the cell. As can be seen from Figure 5, capacitive molecular memory is usually made up of a redoxactive monolayer, which can capture or release electrons, that is attached to the Si substrate. According to the different operations on electrons, the monolayer can be either oxidised (i.e., losing an electron) or reduced (i.e., securing an electron). One or two electrons can escape from the redox-active molecule to bring it to the neutral state and mono- or dipositively charge states, thus assigning the role of chargestorage centre to the molecule. The active monolayer is encapsulated by the dioxide insulator to prevent charge loss, and the electrolyte solution behaving as another barrier is ion-conductive and electron-limited. As a result, the electron released during the oxidation process of the molecule would travel out of the molecule into the substrate, while the electrons in the electrolyte cannot move into the molecule. This would obviously result in a positive charge on the molecule. This positive charge can be reduced by moving the electron back to the molecule from the substrate through a lower gate potential. Therefore, molecular memory can be switched between two different conduction states by the charging and discharging of the cell. Readout is performed by sensing the resistance of the molecular memory cell. At present, several redox-active materials such as ferrocenyl phosphonate (Fc-P) $[57,58]$ and porphyrin-ferrocene (PorFc) [59] have received attentions for widespread applications using capacitive molecular memory owing to their inherent merits such as the nanoscale size, low voltage operation, and multiple state properties. However, the thermal stability problem that causes the degradation of the capacitive molecular device from $100^{\circ} \mathrm{C}$ is currently a formidable challenge that has to be tackled with respect to the capacitive molecular memory technology [60, 61], which needs to be solved in order to move molecular memory from the laboratory level.

The structure of resistive molecular memory usually consists of an organic layer sandwiched by two metal electrodes, similar to macromolecular memory. The recording process is achieved by applying an external voltage that causes a transition of the molecule into one of the two possible resistance states, while readout is realised by measuring the resulting resistance change of the molecular cell. Unlike its capacitive counterpart, the switching mechanisms of resistive molecular memory still remain controversial. It was reported that the conduction behaviour of stearic acid $\left(\mathrm{C}_{18}\right)$ is attributed to the formation of dissolution of individual nanoscale conducting filaments through the molecular layer $[62,63]$. Another hypothesis ascribes the conductance switching of phenylene ethynylene oligomers to the conformational changes in molecules or molecular bundles, which is also likely to be responsible for Ponceau SS [64]. Further clarification on the physical mechanism of resistive molecular memory is urgently required for in-depth analysis of its storage performance.

Compared with other emerging nonvolatile memories, the most appealing property of molecular memory is its outstanding scalability. Because of the small size of the molecule, very dense circuits could be built, and bottomup self-assembly of molecules in complex structures could be applied to augment top-down lithography fabrication techniques. In addition, as all molecules of one type of material are identical, molecular switches would have identical characteristics, thus eliminating the serious problem of variability of components. Because of this key advantage, molecular memory has been regarded as one of the most promising candidates to supplant DRAM, possibly leading to smaller and cheaper integrated circuits as well. Nevertheless, it should be noted that molecular memory also involves some challenges primarily due to the extreme sensitivity of the device characteristics to exterior parameters such as the contacts, reproducible nanogaps, and environment. Moreover, the electrical switching characteristic of the molecular devices still remains ambiguous, in spite of the advent of some plausible interpretations. Because of the above factors, the density of molecular memory is limited to $160 \mathrm{~kb}$ [65]. As a 
TABle 1: Performance comparison of emerging memories.

\begin{tabular}{lcccc}
\hline Attribute & Carbon memory & Mott memory & Macromolecule memory & Molecule memory \\
\hline Switching speed (ns) & $<50$ & $<2.3$ & $<25$ & Relatively slow \\
Endurance & Not tested & Not tested & $>10^{6}$ & Not tested \\
Lifetime (yr) & $>50$ & Not tested & $>1$ & Long \\
Writing energy (per bit) & $\sim \mathrm{pJ}$ & $\sim 100 \mathrm{fJ}$ & $\sim 6 \mathrm{fJ}$ & Not tested \\
\hline
\end{tabular}

consequence, molecular memory is viewed as a long-term research goal, and the field of molecular electronics needs further fundamental work, which is currently in progress $[58,66]$.

\section{Conclusion}

To address the scaling limits challenges that conventional CMOS devices are currently encountering, more advanced nonvolatile memories that can not only reproduce the strength of conventional CMOS memories, but also effectively enhance the storage capacity beyond the scaling limits need to be developed. Some emerging nonvolatile memories including carbon memory, Mott memory, macromolecular memory, and molecular memory have recently gained increased attention because of their immense potential to provide excellent scalability, fast switching time, long retention time, and low power consumption. Table 1 summarises the performance of each of these emerging memories.

Presently, researchers worldwide seem to be more dedicated to carbon-based memories than other emerging types of memories, resulting in a fairly mature technology for system design, fabrication, and optimisation of the former. This is expected as the conduction mechanism of carbonbased materials has been deeply investigated both experimentally and theoretically, and it can be therefore inferred that such a trend would continue in the foreseeable future. At the same time, it has to be noted that other emerging nonvolatile memories have exhibited their respective superiorities with respect to certain aspects that the current carbonbased memories cannot compete with. For example, the switching time of Mott memory, the power consumption of macromolecular memory, and the scalability of molecular memory are comparably better than the corresponding properties of carbon-based memories. As a consequence, numerous successful studies conducted on carbon-based memories have made carbon-based memories seemingly more attractive than alternative memory technologies in the short term. Nevertheless, in the long term the potential of the emerging type of memories can be better estimated when the conduction mechanisms of these newer memories are more clearly understood.

\section{Conflict of Interests}

The authors declare that there is no conflict of interests regarding the publication of this paper.

\section{Acknowledgments}

The authors acknowledge the financial support of the National Natural Science Foundation of China under Grant nos. 61201439 and 61202319, the Educational Bureau of Jiangxi Province Grant no. GJJ13487, and the Key laboratory of Image Processing and Pattern Recognition (Nanchang Hangkong University) Grant no. 20142BAB217013.

\section{References}

[1] S. K. Lai, "Flash memories: successes and challenges," IBM Journal of Research and Development, vol. 52, no. 4.5, pp. 529$535,2008$.

[2] H. C. Chin, C. S. Lim, W. S. Wong et al., "Enhanced device and circuit-level performance benchmarking of graphene nanoribbon field-effect transistor against a Nano-MOSFET with interconnects," Journal of Nanomaterials, vol. 2014, Article ID 879813, 14 pages, 2014.

[3] H. Lan and H. Liu, "UV-nanoimprint lithography: structure, materials and fabrication of flexible molds," Journal of Nanoscience and Nanotechnology, vol. 13, no. 5, pp. 3145-3172, 2013.

[4] H. J. Borg and R. V. Woudenberg, “Trends in optical recording," Journal of Magnetism and Magnetic Materials, vol. 193, no. 1-3, pp. 519-525, 1999.

[5] C.-Y. Lu, "Future prospects of NAND flash memory technology - the evolution from floating gate to charge trapping to 3D stacking," Journal of Nanoscience and Nanotechnology, vol. 12, no. 10, pp. 7604-7618, 2012.

[6] C. Miccoli, C. M. Compagnoni, L. Chiavarone et al., "Reliability characterization issues for nanoscale flash memories: a case study on 45-nm NOR devices," IEEE Transactions on Device and Materials Reliability, vol. 13, no. 2, pp. 362-369, 2013.

[7] S. K. Lai, "Brief history of ETOX ${ }^{\mathrm{U}}$ NOR flash memory," Journal of Nanoscience and Nanotechnology, vol. 12, no. 10, pp. 75977603, 2012.

[8] Y. Fujisaki, "Overview of emerging semiconductor non-volatile memories," IEICE Electronics Express, vol. 9, no. 10, pp. 908-925, 2012.

[9] D. S. Jeong, R. Thomas, R. S. Katiyar et al., "Emerging memories: resistive switching mechanisms and current status," Reports on Progress in Physics, vol. 75, no. 7, Article ID 076502, 2012.

[10] H. Liu, D. Bedau, D. Backes, J. A. Katine, J. Langer, and A. D. Kent, "Ultrafast switching in magnetic tunnel junction based orthogonal spin transfer devices," Applied Physics Letters, vol. 97, no. 24, Article ID 242510, 2010.

[11] T. Kawahara, K. Ito, R. Takemura, and H. Ohno, "Spin-transfer torque RAM technology: review and prospect," Microelectronics Reliability, vol. 52, no. 4, pp. 613-627, 2012.

[12] K. C. Chun, H. Zhao, J. D. Harms, T.-H. Kim, J.-P. Wang, and C. H. Kim, "A scaling roadmap and performance evaluation of 
in-plane and perpendicular MTJ based STT-MRAMs for highdensity cache memory," IEEE Journal of Solid-State Circuits, vol. 48, no. 2, pp. 598-610, 2013.

[13] L. Wang and S. Gai, "The next generation mass storage devices-physical principles and current status," Contemporary Physics, vol. 55, no. 2, pp. 75-93, 2014.

[14] A. Kolobov and M. Popescu, "Phase-change memory: science and applications," Physica Status Solidi (b), vol. 249, no. 10, pp. 1824-1826, 2012.

[15] E. Linn, R. Rosezin, C. Kügeler, and R. Waser, "Complementary resistive switches for passive nanocrossbar memories," Nature Materials, vol. 9, no. 5, pp. 403-406, 2010.

[16] J. J. Yang, M.-X. Zhang, J. P. Strachan et al., "High switching endurance in $\mathrm{TaO}_{x}$ memristive devices," Applied Physics Letters, vol. 97, no. 23, Article ID 232102, 2010.

[17] C. Kugeler, J. Zhang, S. H. Eifert, S. K. Kim, and R. Waser, "Nanostructured resistive memory cells based on 8-nm-thin $\mathrm{TiO}_{2}$ films deposited by atomic layer deposition," Journal of Vacuum Science and Technology B, vol. 29, Article ID 01AD01, 2011.

[18] J. Robertson, "Diamond-like amorphous carbon," Materials Science and Engineering R: Reports, vol. 37, no. 4-6, pp. 129-281, 2002.

[19] J. Robertson, "Amorphous carbon," Advances in Physics, vol. 35, no. 4, pp. 317-374, 1986.

[20] C. Casiraghi, J. Robertson, and A. C. Ferrari, "Diamond-like carbon for data and beer storage," Materials Today, vol. 10, no. 1-2, pp. 44-53, 2007.

[21] J. Xu, D. Xie, T. Feng et al., "Scaling-down characteristics of nanoscale diamond-like carbon based resistive switching memories," Carbon, vol. 75, pp. 255-261, 2014.

[22] B. Standley, W. Bao, Z. Hang, J. Bruck, N. L. Chun, and M. Bockrath, "Graphene-based atomic-scale switches," Nano Letters, vol. 8, no. 10, pp. 3345-3349, 2008.

[23] F. Kreupl, R. Bruchhaus, P. Majewski et al., "Carbon-based resistive memory," in Proceedings of the IEEE International Electron Devices Meeting (IEDM '08), vol. 1, pp. 1-4, San Francisco, Calif, USA, 2008.

[24] F. Zhuge, B. L. Hu, C. L. He, X. F. Zhou, Z. P. Liu, and R. W. Li, "Nonvolatile resistive switching memory based on amorphous carbon," Applied Physics Letters, vol. 96, no. 16, Article ID 163505, 3 pages, 2010.

[25] Y. Chai, Y. Wu, K. Takei et al., "Nanoscale bipolar and complementary resistive switching memory based on amorphous carbon," IEEE Transactions on Electron Devices, vol. 58, no. 11, pp. 3933-3939, 2011.

[26] D. Fu, D. Xie, T. T. Feng et al., "Unipolar resistive switching properties of diamondlike carbon-based RRAM devices," IEEE Xplore: Electron Device Letters, vol. 32, no. 6, pp. 803-805, 2011.

[27] L. Dellmann, A. Sebastian, P. Jonnalagadda et al., "Nonvolatile resistive memory devices based on hydrogenated amorphous carbon," in Proceedings of the European Solid-State Device Research Conference (ESSDERC '13), pp. 268-271, Bucharest, Romania, September 2013.

[28] A. Sebastian, A. Pauza, C. Rossel et al., "Resistance switching at the nanometre scale in amorphous carbon," New Journal of Physics, vol. 13, Article ID 013020, 2011.

[29] S. Qin, J. Zhang, D. Fu et al., "A physics/circuit-based switching model for carbon-based resistive memory with $\mathrm{sp}^{2} / \mathrm{sp}^{3}$ cluster conversion," Nanoscale, vol. 4, no. 20, pp. 6658-6663, 2012.
[30] Y. J. Chen, H. L. Chen, T. F. Young et al., "Hydrogen induced redox mechanism in amorphous carbon resistive random access memory," Nanoscale Research Letters, vol. 9, article 52, 2014.

[31] A. Asamitsu, Y. Tomioka, H. Kuwahara, and Y. Tokura, "Current switching of resistive states in magnetoresistive manganites," Nature, vol. 388, no. 6637, pp. 50-52, 1997.

[32] D. Ruzmetov, G. Gopalakrishnan, J. Deng, V. Narayanamurti, and S. Ramanathan, "Electrical triggering of metal-insulator transition in nanoscale vanadium oxide junctions," Journal of Applied Physics, vol. 106, no. 8, Article ID 083702, 2009.

[33] Y. Zhou, X. Chen, C. Ko, Z. Yang, and S. Ramanathan, "Voltage-triggered ultrafast phase transition in vanadium dioxide switches," IEEE Electron Device Letters, vol. 34, p. 220, 2013.

[34] M. Son, J. Lee, J. Park et al., "Excellent selector characteristics of nanoscale $\mathrm{VO}_{2}$ for high-density bipolar ReRAM applications," IEEE Electron Device Letters, vol. 32, no. 11, pp. 1579-1581, 2011.

[35] S. D. Ha, G. H. Aydogdu, and S. Ramanathan, "Metal-insulator transition and electrically driven memristive characteristics of $\mathrm{SmNiO}_{3}$ thin films," Applied Physics Letters, vol. 98, no. 1, Article ID 012105, 2011.

[36] M. D. Pickett and R. S. Williams, "Sub-100 fJ and subnanosecond thermally driven threshold switching in niobium oxide crosspoint nanodevices," Nanotechnology, vol. 23, Article ID 215202, 2012.

[37] M. D. Pickett, G. Medeiros-Ribeiro, and R. S. Williams, "A scalable neuristor built with Mott memristors," Nature Materials, vol. 12, no. 2, pp. 114-117, 2013.

[38] F. Nakamura, M. Sakaki, Y. Yamanaka, S. Tamaru, T. Suzuki, and Y. Maeno, "Electric-field-induced metal maintained by current of the Mott insulator $\mathrm{C}_{a 2} \mathrm{RuO}_{4}$," Scientific Reports, vol. 3, article 2536, 2013.

[39] K.-H. Xue, C. A. Pazde Araujo, J. Celinska, and C. McWilliams, "A non-filamentary model for unipolar switching transition metal oxide resistance random access memories," Journal of Applied Physics, vol. 109, Article ID 091602, 2011.

[40] J. Celinska, C. McWilliams, C. Paz De Araujo, and K.-H. Xue, "Material and process optimization of correlated electron random access memories," Journal of Applied Physics, vol. 109, no. 9, Article ID 091603, 2011.

[41] P. Stoliar, L. Cario, E. Janod et al., "Universal electric-fielddriven resistive transition in narrow-gap Mott insulators," Advanced Materials, vol. 25, no. 23, pp. 3222-3226, 2013.

[42] V. Guiot, L. Cario, E. Janod et al., "Avalanche breakdown in $\mathrm{GaTa}_{4} \mathrm{Se}_{8-x} \mathrm{Te}_{x}$ narrow-gap Mott insulators," Nature Communications, vol. 4, article 1722, 2013.

[43] M. Nakano, K. Shibuya, D. Okuyama et al., "Collective bulk carrier delocalization driven by electrostatic surface charge accumulation," Nature, vol. 487, no. 7408, pp. 459-462, 2012.

[44] X. Wang, W. Xie, J. Du, C. Wang, N. Zhao, and J.-B. Xu, "Graphene/metal contacts: bistable states and novel memory devices," Advanced Materials, vol. 24, no. 19, pp. 2614-2619, 2012.

[45] H. Matsuzaki, M. Iwata, T. Miyamoto et al., "Excitation-photonenergy selectivity of photoconversions in halogen-bridged pdchain compounds: mott insulator to metal or charge-densitywave state," Physical Review Letters, vol. 113, Article ID 096403, 2014.

[46] P. Stoliar, M. Rozenberg, E. Janod, B. Corraze, J. Tranchant, and L. Cario, "Nonthermal and purely electronic resistive switching in a Mott memory," Physical Review B, vol. 90, Article ID 045146, 2014. 
[47] T. Oka and N. Nagaosa, "Interfaces of correlated electron systems: proposed mechanism for colossal electroresistance," Physical Review Letters, vol. 95, no. 26, Article ID 266403, 4 pages, 2005.

[48] T. Lee and Y. Chen, "Organic resistive nonvolatile memory materials," MRS Bulletin, vol. 37, no. 2, pp. 144-149, 2012.

[49] P. Y. Lai and J. S. Chen, "Electrical bistability and charge transport behavior in Au nanoparticle/poly( $N$-vinylcarbazole) hybrid memory devices," Applied Physics Letters, vol. 93, Article ID 153305, 2008.

[50] A. Carbone, B. K. Kotowska, and D. Kotowski, "Space-chargelimited current fluctuations in organic semiconductors," Physical Review Letters, vol. 95, Article ID 236601, 2005.

[51] C. W. Chu, J. Ouyang, J. H. Tseng, and Y. Yang, "Organic donor-acceptor system exhibiting electrical bistability for use in memory devices," Advanced Materials, vol. 17, pp. 1440-1443, 2005.

[52] W. Bai, R. Huang, Y. Cai, Y. Tang, X. Zhang, and Y. Wang, "Record low-power organic RRAM with Sub-20-nA reset current," IEEE Electron Device Letters, vol. 34, no. 2, pp. 223-225, 2013.

[53] Y. C. Lai, D. Y. Wang, I. S. Huang et al., "Low operation voltage macromolecular composite memory assisted by graphene nanoflakes," Journal of Materials Chemistry C, vol. 1, no. 3, pp. 552-559, 2013.

[54] J. J. Kim, B. Cho, K. S. Kim, T. Lee, and G. Y. Jung, "Electrical characterization of unipolar organic resistive memory devices scaled down by a direct metal-transfer method," Advanced Materials, vol. 23, no. 18, pp. 2104-2107, 2011.

[55] C.-L. Tsai, F. Xiong, E. Pop, and M. Shim, "Resistive random access memory enabled by carbon nanotube crossbar electrodes," ACS Nano, vol. 7, no. 6, pp. 5360-5366, 2013.

[56] K. Asadi, D. M. de Leeuw, B. de Boer, and P. W. M. Blom, "Organic non-volatile memories from ferroelectric phaseseparated blends," Nature Materials, vol. 7, no. 7, pp. 547-550, 2008.

[57] T. Pro, J. Buckley, R. Barattin et al., "From atomistic to device level investigation of hybrid redox molecular/silicon field-effect memory devices," IEEE Transactions on Nanotechnology, vol. 10, no. 2, pp. 275-283, 2011.

[58] T. Pro, J. Buckley, K. Huang et al., "Investigation of hybrid molecular/silicon memories with redox-active molecules acting as storage media," IEEE Transactions on Nanotechnology, vol. 8, no. 2, pp. 204-213, 2009.

[59] Z. Chen, B. Lee, S. Sarkar, S. Gowda, and V. Misra, "A molecular memory device formed by $\mathrm{HfO}_{2}$ encapsulation of redox-active molecules," Applied Physics Letters, vol. 91, Article ID 173111, 2007.

[60] B. De Salvo, J. Buckley, and D. Vuillaume, "Recent results on organic-based molecular memories," Current Applied Physics, vol. 11, no. 2, pp. e49-e57, 2011.

[61] T. Pro, J. Buckley, R. Barattin et al., "Study of ferrocene/silicon hybrid memories: influence of the chemical linkers and device thermal stability," in Proceedings of the 38th European SolidState Device Research Conference (ESSDERC '08), pp. 226-229, Edinburgh, UK, 2008.

[62] C. N. Lau, D. R. Stewart, R. S. Williams, and M. Bockrath, "Direct observation of nanoscale switching centers in metal/molecule/metal structures," Nano Letters, vol. 4, no. 4, pp. 569-572, 2004.
[63] Z. J. Donhauser, B. A. Mantooth, K. F. Kelly et al., "Conductance switching in single molecules through conformational changes," Science, vol. 292, no. 5525, pp. 2303-2307, 2001.

[64] A. Bandyopadhyay and A. J. Pal, "Multilevel conductivity and conductance switching in supramolecular structures of an organic molecule," Applied Physics Letters, vol. 84, no. 6, p. 999, 2004.

[65] J. E. Green, J. Wook Choi, A. Boukai et al., "A 160-kilobit molecular electronic memory patterned at 1011 bits per square centimetre," Nature, vol. 445, no. 7126, pp. 414-417, 2007.

[66] S. P. Cummings, J. Savchenko, and T. Ren, "Functionalization of flat $\mathrm{Si}$ surfaces with inorganic compounds-towards molecular CMOS hybrid devices," Coordination Chemistry Reviews, vol. 255, no. 15-16, pp. 1587-1602, 2011. 

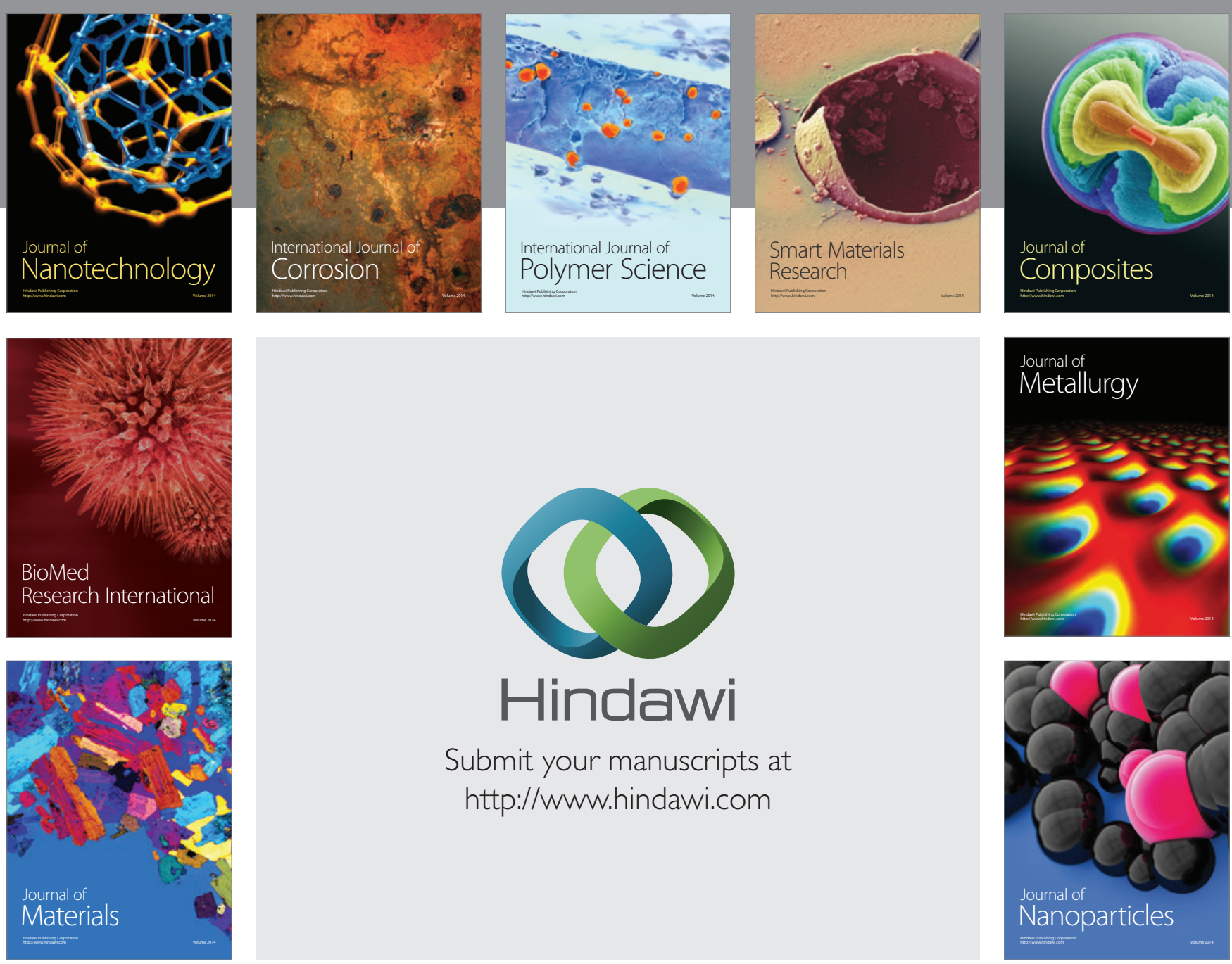

Submit your manuscripts at http://www.hindawi.com
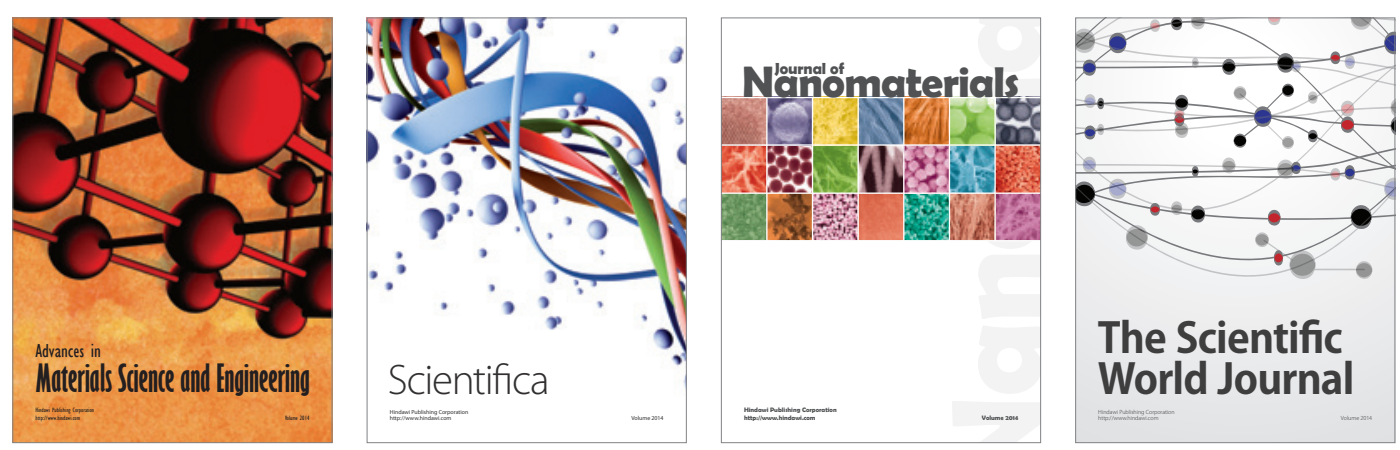

\section{The Scientific World Journal}
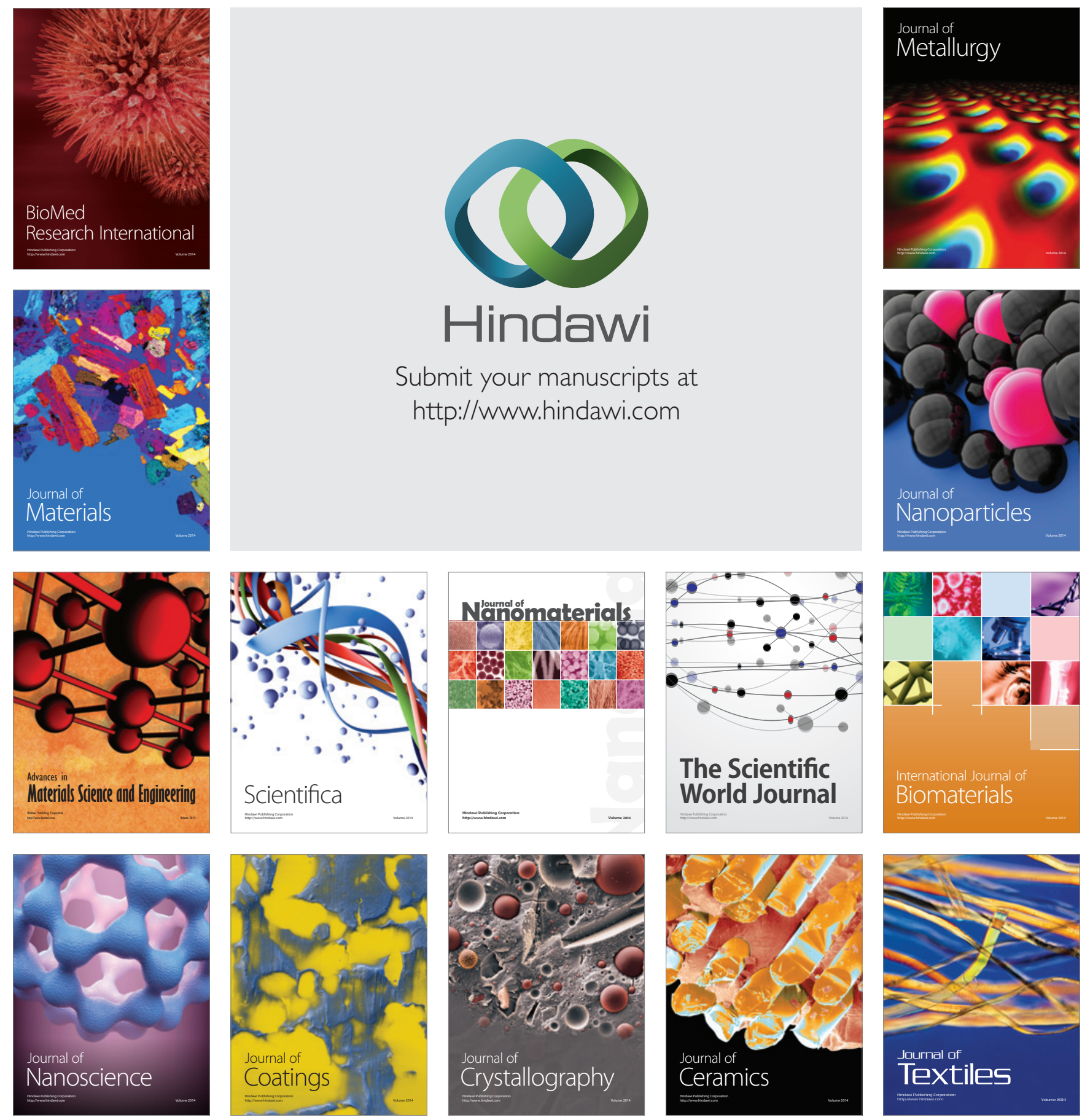